\title{
Computational Complementarity and Shift Spaces ${ }^{1}$
}

\author{
Marjo Lipponen \\ (Department of Mathematics, University of Turku, FIN-20014 Turku, Finland ${ }^{2}$ \\ Email: marlip@utu.fi.)
}

\begin{abstract}
Computational complementarity was introduced to mimic the physical complementarity in terms of finite automata (with outputs but no initial state). Most of the work has been focussed on "frames", i.e., on fixed, static, local descriptions of the system behaviour. The first paper aiming to study the asymptotical description of complementarity was restricted to certain types of sofic shifts. In this paper we continue this work and extend the results to all irreducible sofic shifts. We also study computational complementarity in terms of labelled graphs rather than automata.
\end{abstract}

Key Words: Complementarity principles, finite automata, sofic shifts, graphs.

Category: Category F.1.1 - Models of Computation

\section{Motivation}

Finite automata (with outputs but no initial states) have been extensively used as models of computational complementarity, a property which mimics the physical complementarity. All this work (e.g. Moore [12], Conway [7], Finkelstein and Finkelstein [8], Svozil [14], Calude, Calude, Svozil, Yu [3], Calude, Calude and Khoussainov [2], Calude and Lipponen [6]) was focussed on "frames", i.e., on fixed, static, local descriptions of the system behaviour.

In Calude and Lipponen [4] we took another view as we were mainly interested in the asymptotical description of complementarity. We studied the asymptotical behaviour of two complementarity principles - motivated by Moore's work [12] (see also Conway [7, p. 21] and Svozil [14]) and introduced by Calude, Calude, Svozil, Yu [3] — by associating to every incomplete deterministic automaton (with output, but no initial state) - studied in Calude and Lipponen [6] - certain sofic shifts.

In this paper we continue this research by extending the results to larger class of sofic shifts. We will prove that there is a strong relation between "local complementarity", as it is perceived at the level of "frames", and "asymptotical complementarity" as it is described by the irreducible sofic shift, as graphs having the same behaviour correspond to a unique sofic shift. To this aim we define the notion of complementarity properties to graphs but are also able to find a connection to finite automata.

\section{Notations}

If $S$ is a finite set, then $|S|$ denotes the cardinality of $S$. A partial function $f: A \stackrel{\circ}{\rightarrow} B$ is a function defined for some elements from $A$. In case $f$ is not

\footnotetext{
${ }^{1}$ C. S. Calude and G. Ştefănescu (eds.). Automata, Logic, and Computability. Special issue dedicated to Professor Sergiu Rudeanu Festschrift.

${ }^{2}$ The work was partially done when the author visited Department of Computer Science, The University of Auckland.
} 
defined on $a \in A$ we write $f(a)=\infty$. Let $D(f)=\{a \in A \mid f(a) \neq \infty\}$ denote the domain of $f$. If $D(f)=A$, we say that $f$ is total. Two partial functions $f$ and $g$ are equal, when $D(f)=D(g)$ and $f(a)=g(a)$, for every $a \in D(f)$. For any two sets $A$ and $B$, we denote their symmetrical difference by $\triangle, A \triangle B=(A \backslash B) \cup(B \backslash A)$. If $\Sigma$ is a finite set, called alphabet, then $\Sigma^{*}$ stands for the set of all finite words over $\Sigma$ and the empty word, denoted by $\lambda$, whereas $\Sigma^{\mathbf{Z}}$ is the set of all bi-infinite words over $\Sigma$. An element of $\Sigma^{\mathbf{Z}}$ is a sequence $x=\left(x_{n}\right)_{n \in \mathbf{Z}}=\ldots x_{-1} x_{0} x_{1} \ldots$

A (labeled) graph is a triple $G=\left(S_{G}, \mathcal{E}, \mathcal{L}\right)$, where $\mathcal{E}$ is an edge set, $S_{G}$ the vertex set, and the labeling $\mathcal{L}: \mathcal{E} \rightarrow \Sigma$ assigns to each edge $e$ of $G$ a label $\mathcal{L}(e)$ from the finite alphabet $\Sigma$.

A graph is right-resolving if for each vertex $p \in S_{G}$ the edges starting from $p$ carry different labels. Since we are only dealing with right-resolving graphs in this article, instead of using the labeling function $\mathcal{L}$ we will borrow a notion of transition function from automata theory. So every (right-resolving) graph is a pair $\left(S_{G}, \Delta_{G}\right)$ where $\Delta_{G}$ is a partial function from the set of vertices $S_{G}$ and the labels $\Sigma$ to the set of vertices $S_{G}$. For instance, $\Delta_{G}(p, a)=q$ means that there is an edge labeled by $a$ from the vertex $p$ to the vertex $q$. And if $\Delta_{G}(p, b)=\infty$ then there is no edge with the label $b$ starting from the vertex $p$. The transition diagram $\Delta_{G}$ can be naturally extended to a partial function, $\Delta_{G}: S_{G} \times \Sigma^{*} \stackrel{\circ}{\rightarrow} S_{G}$ as follows: for every $p \in S_{G}, w \in \Sigma^{*}$ and $\sigma \in \Sigma$, $\Delta_{G}(p, \lambda)=p$, and $\Delta_{G}(p, \sigma w)=\Delta_{G}\left(\Delta_{G}(p, \sigma), w\right)$ if $\Delta_{G}(p, \sigma) \neq \infty$. Hence rightresolving graphs have a "deterministic behaviour" in the sense that for every word $w \in \Sigma^{*}$ and every vertex $p$, there is at most one path starting from $p$ which is labeled with $w$.

For all $p \in S_{G}$, the follower set $\mathcal{F}_{G}(p)=\left\{w \in \Sigma^{*} \mid \Delta(p, w) \neq \infty\right\}$ consists of all sequences of labels of paths from the vertex $p$ to any other vertex $q \in S_{G}$.

Further on, we say that a graph $G=(S, \Delta)$ is strongly connected if for every pair of vertices $p, q \in S$ there is a word $w \in \mathcal{F}(p)$ such that $\Delta(p, w)=q$.

Follower sets naturally define how a graph $G_{1}$ can be thought to be simulated by another graph $G_{2}$ meaning that any vertex $p$ of $G_{1}$ has a corresponding (not necessarily unique) vertex $q \in G_{2}$ such that the paths starting from $p$ have exactly the same labels as the paths starting from $q$. Formally, a graph $G_{1}=\left(S_{1}, \Delta_{1}\right)$ is $\mathcal{F}$-simulated by a graph $G_{2}=\left(S_{2}, \Delta_{2}\right)$ if there is a mapping $h: S_{1} \rightarrow S_{2}$ such that $\mathcal{F}_{G_{1}}(p)=\mathcal{F}_{G_{2}}(h(p))$ for all $p \in S_{1}$. If $G_{1}$ and $G_{2}$ are both $\mathcal{F}$-simulating each other, we say that $G_{1}$ and $G_{2}$ are $\mathcal{F}$-equivalent. If, moreover, the mapping $h: S_{1} \rightarrow S_{2}$ is one-to-one and onto, then $G_{1}$ and $G_{2}$ are isomorphic. Notice that this isomorphism is only between vertices. The usual labeled-graph isomorphism is between edges (and labels) as well. But for our purposes in this article the first type is sufficient.

A graph $G_{1}$ is minimal if every graph $G_{2}$ which is $\mathcal{F}$-equivalent to $G_{1}$ has at least as many vertices as $G_{1},\left|S_{1}\right| \leq\left|S_{2}\right|$.

\section{Computational Complementarity}

We say that two vertices $p, q \in S$ in a graph $G=\left(S_{G}, \Delta_{G}\right)$ are indistinguishable if they have the same follower sets, $\mathcal{F}_{G}(p)=\mathcal{F}_{G}(q)$. Hence, if there is a nonempty word $w$ in the set $\mathcal{F}_{G}(p) \triangle \mathcal{F}_{G}(q)$ we say that $p$ and $q$ are distinguishable by $w$. 
Using this definition, we extend the notions of the properties $\mathbf{A}, \mathbf{B}, \mathbf{C}$ which were introduced for automata (with outputs) in Calude, Calude, Svozil, Yu [3] to cover graphs. A graph $G$ has property $\mathbf{A}$ if every pair of its distinct vertices are distinguishable. $G$ has $\mathbf{B}$ if for every vertex $p$ of $G$ there exists a word which distinguishes $p$ from all the other vertices. Finally, $G$ has $\mathbf{C}$ if there exists a word which distinguishes between any two distinct vertices of $G$.

Further on, $G$ satisfies a complementarity principle $C I$ if it has $\mathbf{A}$ but not $\mathbf{B}$ and $C I I$ if it has $\mathbf{B}$ but not $\mathbf{C}$.
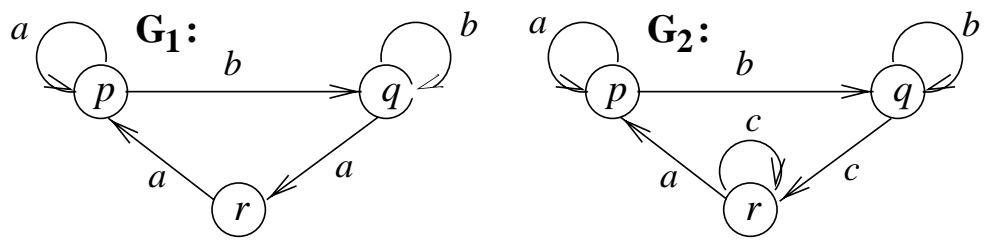

Figure 1: A graph $G_{1}$ satisfies principle $C I$ and $G_{2}$ satisfies $C I I$.

Example 1. The graph $G_{1}$ presented in Figure 1 has property $\mathbf{A}$ since the vertices $p$ and $r$ are distinguishable by $w=b$ and $p$ and $q$ by $w=a b$. But $G_{1}$ does not have $\mathbf{B}$ since there is no word which distinguishes the vertex $p$ from the other vertices. Any word starting with $a$ belongs to both $\mathcal{F}_{G}(p)$ and $\mathcal{F}_{G}(r)$ and any word starting with $b$ belongs to $\mathcal{F}_{G}(p) \cap \mathcal{F}_{G}(r)$.

The graph $G_{2}$ has property $\mathbf{B}$ since the words $a, b$ and $c$ distinguish the vertices $q, r$ and $p$, respectively, from the other vertices. On the other hand, any word starting with $a$ cannot distinguish between $p$ and $r$, with $b$ cannot distinguish between $p$ and $q$ and with $c$ cannot distinguish between $q$ and $r$, respectively. Hence $G_{2}$ does not have $\mathbf{C}$.

Compared to automata with outputs, graphs turn out to be more restricted in terms of complementarity properties.

Proposition 1. Let $G=\left(S_{G}, \Delta_{G}\right)$ be a graph. If $\left|S_{G}\right| \geq 3$ then $G$ cannot have property $\mathbf{C}$.

Proof. Let $p, q, r \in S$ be three distinct vertices. If there is a word $w$ which can distinguish between all of them and $w \in \mathcal{F}_{G}(p) \backslash \mathcal{F}_{G}(q)$ then $w \in \mathcal{F}_{G}(p) \backslash \mathcal{F}_{G}(r)$, and hence, $w \notin \mathcal{F}_{G}(q) \cup \mathcal{F}_{G}(r)$, a contradiction.

Lemma 2. Isomorphism preserves properties $\mathbf{A}, \mathbf{B}$ and $\mathbf{C}$.

Proof. Since isomorphism preserves the follower sets, also the properties A, $\mathbf{B}$ and $\mathbf{C}$ are invariant.

The relation of indistinguishability $\equiv$ partitions the vertex set of a graph $G$ into disjoint equivalence classes. Let $[s]$ denote the class of the vertex $s \in S_{G}$, $[s]=\left\{p \in S_{G} \mid p \equiv s\right\}$. The merged graph $M(G)=\left(S_{M}, \Delta_{M}\right)$ can be defined by setting $S_{M}=\left\{[s] \mid s \in S_{G}\right\}$ and $\Delta_{M}([s], a)=[r]$ if there are vertices $r, s \in S_{G}$ such that $\Delta_{G}(p, a)=q$. 
Theorem 3. Let $G$ be a graph. Then

1) $M(G)$ has property $\mathbf{A}$.

2) $M(G)$ and $G$ are $\mathcal{F}$-equivalent.

3) $M(G)$ is minimal.

Proof. 1) The merged graph $M(G)$ has property $\mathbf{A}$ by the definition.

2) Notice first that for each class $[s]$ we can fix a unique representative to be the vertex $s_{\text {rep }} \in S_{G}$ having, say, the minimum index between all the vertices in the same class. Thus the mapping $h: S_{M} \rightarrow S_{G}, h([s])=s_{\text {rep }}$ is well-defined and for all $[s] \in S_{M}, \mathcal{F}([s])=\mathcal{F}\left(s_{\text {rep }}\right)$ by the definition. Hence $M(G)$ is $\mathcal{F}$ simulated by $G$ via $h$; on the other hand, $G$ is $\mathcal{F}$-simulated by $M(G)$ via the natural mapping $g: S_{G} \rightarrow S_{M}, g(s)=[s]$.

3) Let $B=\left(S_{B}, \Delta_{B}\right)$ be a graph which is $\mathcal{F}$-equivalent to $G$ via the mappings $h_{1}: S_{G} \rightarrow S_{B}$ and $h_{2}: S_{B} \rightarrow S_{G}$. Consider the mapping $l: S_{B} \rightarrow S_{M}$ defined by $l(s)=\left[h_{2}(s)\right]$. Since $s \equiv h_{2}\left(h_{1}(s)\right)$ for all $s \in S_{G}$, and $t \equiv h_{1}\left(h_{2}(t)\right)$ for all $t \in S_{B}$, it follows that for any $[q] \in S_{M}$, there is a vertex $h_{1}(q) \in S_{B}$ such that $l\left(h_{1}(q)\right)=\left[h_{2}\left(h_{1}(q)\right)\right]=[q]$. So $l$ is an onto function from the finite set $S_{B}$ to the finite set $S_{M}$, and hence, $\left|S_{M}\right| \leq\left|S_{B}\right|$.

Theorem 4. Two merged graphs $G_{1}$ and $G_{2}$ are $\mathcal{F}$-equivalent iff they are isomorphic.

Proof. Assume first that $G_{1}$ and $G_{2}$ are $\mathcal{F}$-equivalent via the mappings $h_{1}: S_{1} \rightarrow S_{2}$ and $h_{2}: S_{2} \rightarrow S_{1}$. By Theorem 3, $\left|S_{1}\right|=\left|S_{2}\right|$. Indeed, since $G_{1}=M\left(G_{1}\right)$ and $G_{2}=M\left(G_{2}\right)$, we have $\left|S_{1}\right| \leq\left|S_{2}\right|$ and $\left|S_{2}\right| \leq\left|S_{1}\right|$. On the other hand, since all the states in $G_{1}$ and $G_{2}$ are distinguishable this implies that the mappings $h_{1}$ and $h_{2}$ are isomorphisms. The second part of the statement follows from the definition.

Corollary 5. Two graphs $G_{1}$ and $G_{2}$ are $\mathcal{F}$-equivalent iff their merged graphs $M\left(G_{1}\right)$ and $M\left(G_{2}\right)$ are isomorphic.

\section{Sofic shifts}

A sofic shift $X$ is a subset of $\Sigma^{\mathbf{Z}}$ consisting of all bi-infinite walks (sequences of labels) on some graph $G$. We say that $G$ is a presentation of $X$, and we write $X=X_{G}$. By a well-known result (see Lind and Marcus [11]) every sofic shift $X$ has a right-resolving presentation. The language of a shift $X$ is the set $\mathcal{B}(X)$ of all subwords of sequences in $X$.

In this section we will consider mainly strongly connected graphs which correspond irreducible sofic shifts. Recall that a shift $X$ is called irreducible if for all words $u, v \in \mathcal{B}(X)$ there is a word $w \in \mathcal{B}(X)$ such that $u w v \in \mathcal{B}(X)$. By Lind and Marcus [11], the shift $X_{G}$ is irreducible iff its presentation $G$ is strongly connected.

A minimal (right-resolving) presentation of a sofic shift $X$ is a presentation which has the fewest number of vertices among all (right-resolving) 
presentations of $X$. A minimal presentation coincides with a minimal graph and is unique up to an isomorphism as the following results will show. Our aim in this section is to characterize all the right-resolving presentations corresponding to the same irreducible sofic shift.

The first result, see Lind and Marcus [11], shows that if $G$ is a presentation of a shift space $X$ then also its merged graph $M(G)$ is a presentation of $X$.

Proposition 6. For a graph $G, X_{G}=X_{M(G)}$. Furthermore, if $G$ is irreducible (resp. right-resolving), then so is $M(G)$.

The following two results are due to Fischer $[9,10]$.

Proposition 7. Any two minimal right-resolving presentations of an irreducible sofic shift are isomorphic as labeled graphs.

Proposition 8. Let $X$ be an irreducible sofic shift and $G$ its irreducible rightresolving presentation. Then the merged graph $M(G)$ is the minimal rightresolving presentation of $X$.

We are now ready to prove our main result.

Theorem 9. Two graphs $G_{1}$ and $G_{2}$ are $\mathcal{F}$-equivalent iff $X_{G_{1}}=X_{G_{2}}$.

Proof. If $G_{1}$ and $G_{2}$ are $\mathcal{F}$-equivalent then $M\left(G_{1}\right)$ and $M\left(G_{2}\right)$ are isomorphic by Corollary 5 . But this implies that $X_{M\left(G_{1}\right)}=X_{M\left(G_{2}\right)}$, and hence by Proposition $6, X_{G_{1}}=X_{G_{2}}$.

On the other hand, if $X_{G_{1}}=X_{G_{2}}$ then by Propositions 7 and $8, M\left(G_{1}\right)$ and $M\left(G_{2}\right)$ are isomorphic and again by Corollary $5, G_{1}$ and $G_{2}$ are $\mathcal{F}$-equivalent.

Theorem 9 can be used to express complementarity principles in terms of properties of sofic shifts.

Corollary 10. Let $G_{1}$ and $G_{2}$ be two graphs. If $G_{1}$ satisfies $C I$ and $G_{2}$ satisfies CII, then the sofic shifts $X_{G_{1}}$ and $X_{G_{2}}$ cannot be equal.

Corollary 11. If $G_{1}$ and $G_{2}$ are merged graphs presenting the same shift, $X_{G_{1}}=$ $X_{G_{2}}$, then they satisfy the same principle CI/CII.

Example 2. Notice that Theorem 9 is not valid for graphs which are not strongly connected. Indeed, the graphs $G_{1}$ and $G_{2}$ in Figure 2 are presentations of the same sofic shifts but are not $\mathcal{F}$-equivalent. Furthermore, $G_{1}$ has property $\mathbf{C}$ while $G_{2}$ satisfies principle $C I I$.

\section{Incomplete automata}

In this section we will approach the sofic shifts from another point of view by studying the theory of incomplete automata introduced in Calude and Lipponen [6]. 

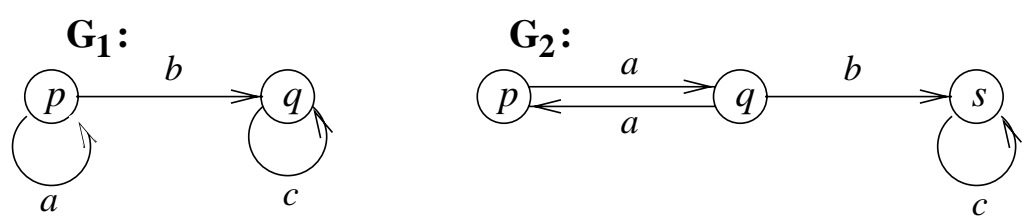

Figure 2: $G_{1}$ and $G_{2}$ generate the same shift but are not $\mathcal{F}$-equivalent.

An incomplete automaton can be seen as a labeled graph with an extension that every vertex produces an output. Formally, a deterministic (finite) incomplete automaton over the alphabets $\Sigma$ (input symbols) and $O$ (output symbols) is a triple $A=\left(S_{A}, \Delta_{A}, F_{A}\right)$, where the set of states $S_{A}$ is finite and nonempty, the transition table $\Delta_{A}$ is a partial function from $S_{A} \times \Sigma$ to the set of states $S_{A}$, and the output function $F_{A}$ is a (total) mapping from the set of states $S_{A}$ into the output alphabet $O$. The graph $G_{A}=\left(S_{A}, \Delta_{A}\right)$ is said to be the underlying graph of the automaton $A$.

The counterpart of the follower set in a graph is the set of applicable words in an automaton. For all $p \in S_{A}$, the set $W_{A}(p)=\left\{w \in \Sigma^{*} \mid \Delta_{A}(p, w) \neq \infty\right\}$ consists of all words leading to complete computations on state $p$. Furthermore, an automaton $A=\left(S_{A}, \Delta_{A}, F_{A}\right)$ is strongly connected if the underlying graph $G_{A}$ is strongly connected, that is, for every pair of states $p, q \in S_{A}$ there is a word $w \in W_{A}(p)$ such that $\Delta_{A}(p, w)=q$.

The response of an automaton $A=\left(S_{A}, \Delta_{A}, F_{A}\right)$ to an input signal is a partial function $R_{A}: S_{A} \times \Sigma^{*} \stackrel{\circ}{\rightarrow} O^{*}$ defined such that for every $s \in S_{A}$, $R_{A}(s, \lambda)=F_{A}(s)$, and

$R_{A}\left(s, \sigma_{1} \ldots \sigma_{n}\right)=F_{A}(s) F_{A}\left(\Delta_{A}\left(s, \sigma_{1}\right)\right) F_{A}\left(\Delta_{A}\left(s, \sigma_{1} \sigma_{2}\right)\right) \ldots F_{A}\left(\Delta_{A}\left(s, \sigma_{1} \ldots \sigma_{n}\right)\right)$, if $\sigma_{1} \ldots \sigma_{n} \in W_{A}(s), \sigma_{i} \in \Sigma, n \geq 1$ and $1 \leq i \leq n$.

\section{A:}

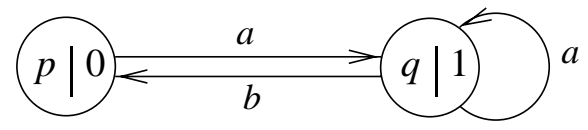

Figure 3: An automaton with two states.

Example 3. Let $\Sigma=\{a, b\}, O=\{0,1\}$, and consider the strongly connected two-state automaton $A$ presented in Figure 3 . The state $p$ emits an output 0 , $F_{A}(p)=0$, and the state $q$ emits an output $1, F_{A}(q)=1$. The responses to an input $a b$ are $R_{A}(p, a b)=010, R_{A}(q, a b)=110$, and to an input $b a, R_{A}(p, b a)=$ $\infty, R_{A}(q, b a)=101$.

In automata theory $\beta$-simulation comes from behavioral simulation, meaning that an automaton can perform all computations performed by another automaton and produces the same outputs. We say that an automaton 
$A=\left(S_{A}, \Delta_{A}, F_{A}\right)$ is $\beta$-simulated by an automaton $B=\left(S_{B}, \Delta_{B}, F_{B}\right)$ if there is a mapping $h: S_{A} \rightarrow S_{B}$ such that for all $s \in S_{A}, W_{A}(s)=W_{B}(h(s))$ and $R_{A}(s, w)=R_{B}(h(s), w)$, for all $w \in W_{A}(s)$. Correspondingly, if $A$ and $B$ both $\beta$-simulate each other, we say that they are $\beta$-equivalent, and if the mapping $h: S_{A} \rightarrow S_{B}$ is one-to-one and onto, and for all $s \in S_{A}$ and $\sigma \in W_{A}(s) \cap \Sigma$, $h\left(\Delta_{A}(s, \sigma)\right)=\Delta_{B}(h(s), \sigma)$, then $A$ and $B$ are isomorphic. An automaton $A$ is minimal if every automaton $B$ which is $\beta$-equivalent to $A$ has at least as many states as $A,\left|S_{A}\right| \leq\left|S_{B}\right|$.

Two states $p, q \in S_{A}$ are indistinguishable iff $W_{A}(p)=W_{A}(q)$ and $R_{A}(p, w)=R_{A}(q, w)$ for all $w \in W_{A}(p)$. If $p$ and $q$ are not indistinguishable we say that they are distinguishable and every word from the set

$$
\left\{w \mid w \in W_{A}(p) \triangle W_{A}(q) \text { or } R_{A}(p, w) \neq R_{A}(q, w)\right\}
$$

is said to distinguish between $p$ and $q$. Using this definition, we define properties $\mathbf{A}, \mathbf{B}$ and $\mathbf{C}$ as well as principles $C I$ and $C I I$ in the same way as for the graphs in Section 3.

In the previous section we considered sofic shifts which are a special class of shift spaces. Formally, a subset $X$ of $\Sigma^{\mathbf{Z}}$ is a shift space if it is topologically closed (with respect to the natural metric on $\Sigma^{\mathbf{Z}}$ ) and shift invariant, $\sigma(X)=X$, where $\sigma: \Sigma^{\mathbf{Z}} \rightarrow \Sigma^{\mathbf{Z}}$ is a shift transformation $\sigma(x)_{i}=x_{i+1}$. The set $\Sigma^{\mathbf{Z}}$ is called the full shift. Two shift spaces $X$ and $Y$ are conjugate if there is a oneto-one onto morphism $\phi: X \rightarrow Y$ which commutes with the shift transformation, $\phi \circ \sigma_{X}=\sigma_{Y} \circ \phi$. For more details, see Lind and Marcus [11].

Following Calude and Lipponen [4] we associate the label-output shift (of bi-infinite sequences) to each automaton $A=\left(S_{A}, \Delta_{A}, F_{A}\right)$ :

$$
\mathcal{S}_{A}^{\Sigma, O}=\left\{\left(a_{i}, x_{i}\right)_{i \in \mathbf{Z}} \mid q_{i} \in S_{A}, a_{i} \in \Sigma, x_{i} \in O, \Delta_{A}\left(q_{i}, a_{i}\right)=q_{i+1}, x_{i}=F_{A}\left(q_{i}\right)\right\} .
$$

Other relations between finite automata (with initial states) and sofic shifts were explored by various authors; see Béal and Perrin [1], Perrin [13].

In Calude and Lipponen [4] we proved that every label-output shift is a sofic shift by introducing for each strongly connected automaton $A$ a corresponding graph $G_{A}$ such that $\mathcal{S}_{A}^{\Sigma, O}=X_{G_{A}}$. The following example shows the opposite situation, a way to find an automaton which corresponds to the given graph.

G:

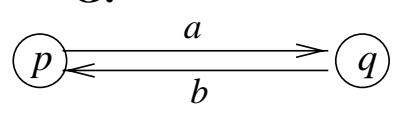

A:

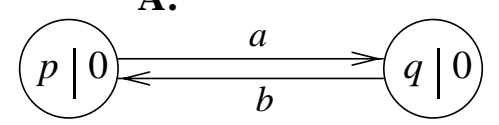

Figure 4: The label-output shift $\mathcal{S}_{A}^{\Sigma, O}$ and the sofic shift $X_{G}$ correspond to each other.

Example 4. Consider the graph $G$ and the automaton $A$ presented in Figure 4. We notice that the sequences running through the first coordinates in the labeloutput shift $\mathcal{S}_{A}^{\Sigma, O}$ make the sofic shift $X_{G}$. 
Formally, let $G=\left(S_{G}, \Delta_{G}\right)$ be a graph over the alphabet $\Sigma$. The automaton $A_{G}=\left(S_{A}, \Delta_{A}, F_{A}\right)$ has $G$ as its underlying graph and we let every state $p \in S_{A}$ emit an output $0, F(p)=0$. It follows immediately that $a \in W_{A}(p)$ iff $a \in \mathcal{F}_{G}(p)$.

Hence we can find for any sofic shift $X$ a corresponding label-output shift $\mathcal{S}_{A}^{\Sigma, O}$ where $O=\{0\}$ and $A=A_{G}$ for some right-resolving presentation $G$ of $X$ such that $X=h\left(\mathcal{S}_{A}^{\Sigma, O}\right)$ where $h:(\Sigma \times O)^{*} \rightarrow \Sigma$ is defined by $h(a, 0)=a$ for all $a \in \Sigma$.

The automaton $A_{G}$ is strongly connected and deterministic iff the graph $G$ is strongly connected and right-resolving. In what follows, we consider only rightresolving graphs; however, we do not assume them to be strongly connected.

Theorem 12. Two states $p, q \in S_{A_{G}}$ are indistinguishable iff $\mathcal{F}_{G}(p)=\mathcal{F}_{G}(q)$.

Proof. If the states $p, q$ in $A_{G}$ are indistinguishable then $W_{A}(p)=W_{A}(q)$ and $R_{A}(p, w)=R_{A}(q, w)$ for all $w \in W_{A}(p)$. Since the output alphabet of $A_{G}$ consists of only one letter, all the responses are just sequences of this letter. So two states in $A_{G}$ are indistinguishable iff $W_{A}(p)=W_{A}(q)$. But this means that in the graph $G$, the follower sets of the vertices $p$ and $q$ are the same, $\mathcal{F}_{G}(p)=\mathcal{F}_{G}(q)$.

In the same way we prove the other implication.

For an incomplete automaton $A=\left(S_{A}, \Delta_{A}, F_{A}\right)$ the length of the shortest words to check whether two states $p, q \in S_{A}$ are distinguishable is $\left|S_{A}\right|-1$ (see Calude and Lipponen [6]). With this in mind we are able to improve the bound presented in Lind and Marcus [11] for right-resolving graphs.

Theorem 13. The shortest word needed to check whether two vertices in graph $G=(S, \Delta)$ are distinguishable is of length $|S|-1$.

Notice that Example 1 shows that this limit cannot be further improved. Indeed, the shortest word to distinguish between the vertices $p$ and $q$ in $G_{1}$ is $a b$ and $|a b|=\left|S_{1}\right|-1$.

Theorem 14. The automaton $A_{G}$ has property $\mathbf{A}$ (resp. B or $\mathbf{C}$ ) iff $G$ has property $\mathbf{A}$ (resp. B or $\mathbf{C})$.

Proof. Follows from the relation $w \in W_{A}(p)$ iff $w \in \mathcal{F}_{G}(p)$ for all $p \in S_{G}$.

In Calude and Lipponen [6] we constructed an algorithm which decides whether an automaton has properties $\mathbf{A}, \mathbf{B}$ or $\mathbf{C}$.

Corollary 15. The properties $\mathbf{A}, \mathbf{B}$ and $\mathbf{C}$ are decidable for graphs.

\section{References}

[1] Marie-Pierre Béal and Dominique Perrin. Symbolic dynamics and finite automata, In G. Rozenberg and A. Salomaa, editors, Handbook of Formal Languages, Vol. 2, pages 463-506. Springer-Verlag, Heidelberg, 1997. 
[2] C. Calude, E. Calude, and B. Khoussainov. Deterministic automata: simulation, universality and minimality. Annals of Pure and Applied Logic, 90, 1-3: 263-276, 1997.

[3] C. Calude, E. Calude, K. Svozil, and S. Yu. Physical versus computational complementarity I. International Journal of Theoretical Physics, 36:1495-1523, 1997.

[4] C. S. Calude and M. Lipponen. Computational complementarity and sofic shifts. In X. Lin, editor, Computing Theory '98, pages 277-290. Springer, Singapore, 1998.

[5] E. Calude and M. Lipponen. Minimal deterministic incomplete automata. Journal of Universal Computer Science, 3(11):131-149, 1997.

[6] E. Calude and M. Lipponen. Deterministic incomplete automata: simulation, universality and complementarity. In C. S. Calude, J. Casti, and M. J. Dinneen, editors, Unconventional Models of Computation, pages 131-149. Springer-Verlag, Singapore, 1998.

[7] J. H. Conway. Regular Algebra and Finite Machines. Chapman and Hall Ltd., London, 1971.

[8] D. Finkelstein and S. R. Finkelstein. Computational complementarity. International Journal of Theoretical Physics, 22(8):753-779, 1983.

[9] R. Fischer. Sofic systems and graphs. Monatshefte für Mathematik, 80(3):179-186, 1975.

[10] R. Fischer. Graphs and symbolic dynamics. In I. Csiszár and P. Elias, editors, Topics in Information Theory 16, pages 229-243. North-Holland, Budapest, 1975.

[11] D. Lind and B. Marcus. Symbolic Dynamics and Coding, Cambdridge University Press, 1995.

[12] E. F. Moore. Gedanken-experiments on sequential machines. In C. E. Shannon and J. McCarthy, editors, Automata Studies, pages 128-153. Princeton University Press, Princeton, 1956.

[13] D. Perrin. Symbolic dynamics and finite automata. In J. Wiedermann and P. Hájek, editors, Proc. 20th International Symposium MFCS'95, pages 94-104. Springer-Verlag, Heidelberg, 1995.

[14] Karl Svozil. Randomness $\&$ Undecidability in Physics. World Scientific, Singapore, 1993. 